\title{
Pneumatose Cólica Tratada com Metronidazol e Oxigenoterapia Hiperbárica: Um Caso de Sucesso
}

\author{
Pneumatosis Coli Treated with Metronidazole and Hyperbaric Oxygen \\ Therapy: A Successful Case
}

\author{
Mariana COSTA ${ }^{1}$, Carolina MORGADO ${ }^{2}$, David ANDRADE² ${ }^{2}$ Francisco GUERREIRO ${ }^{3}$, João COIMBRA $^{1}$ \\ Acta Med Port 2015 Jul-Aug;28(4):534-537
}

\section{RESUMO}

A pneumatose intestinal, caracterizada pela presença de gás na parede do cólon, é uma condição incomum com um espectro de apresentação variável. Pode ser idiopática ou secundária a outras doenças. A tomografia computorizada é o método de diagnóstico com maior sensibilidade. Na ausência de sinais e sintomas sugestivos de complicações, como a perfuração e a peritonite, a pneumatose intestinal pode ser tratada de forma conservadora. Apresenta-se o caso de uma mulher de 59 anos com pneumatose cólica secundária a teratoma ovárico benigno. Após a cirurgia, manteve-se sintomática e foi tratada com sucesso com metronidazol e oxigenoterapia hiperbárica.

Palavras-chave: Metronidazol; Oxigenação Hiperbárica; Pneumatose Cistoide Intestinal.

\section{ABSTRACT}

Pneumatosis intestinalis, characterized by the presence of gas within the bowel wall, is an uncommon condition with variable presentation. It may be idiopathic or secondary to other diseases. A computed tomography scan is the most sensitive method for diagnosis. In the absence of signs and symptoms of complications, such as perforation and peritonitis, pneumatosis intestinalis can be managed conservatively. We present the case of a 59-year-old woman with pneumatosis coli secondary to benign ovary teratoma. After surgery she remained symptomatic and was successfully treated with metronidazole and hyperbaric oxygen therapy.

Keywords: Hyperbaric Oxygenation; Metronidazole; Pneumatosis Cystoides Intestinalis.

\section{INTRODUÇÃO}

A pneumatose cólica (PC), um subtipo da pneumatose intestinal $(\mathrm{PI})$, caracteriza-se pela presença de múltiplos quistos na parede do cólon contendo nitrogénio, hidrogénio e dióxido de carbono. As formações quísticas podem estar confinadas à mucosa, submucosa ou serosa; podendo envolver as três camadas. ${ }^{1}$ Apesar da tomografia computorizada (TC) ser a ferramenta diagnóstica com maior sensibilida- de, a PC pode ser encontrada acidentalmente durante uma colonoscopia. Na maioria dos casos está associada a outra patologia, tendo um largo espectro de apresentação. Expõe-se a história de uma doente com PC associada a teratoma ovárico que manteve sintomas e áreas cólicas com formações gasosas após a ressecção cirúrgica do tumor e que foi tratada com antibióticos e oxigenoterapia hiperbárica (OHB).
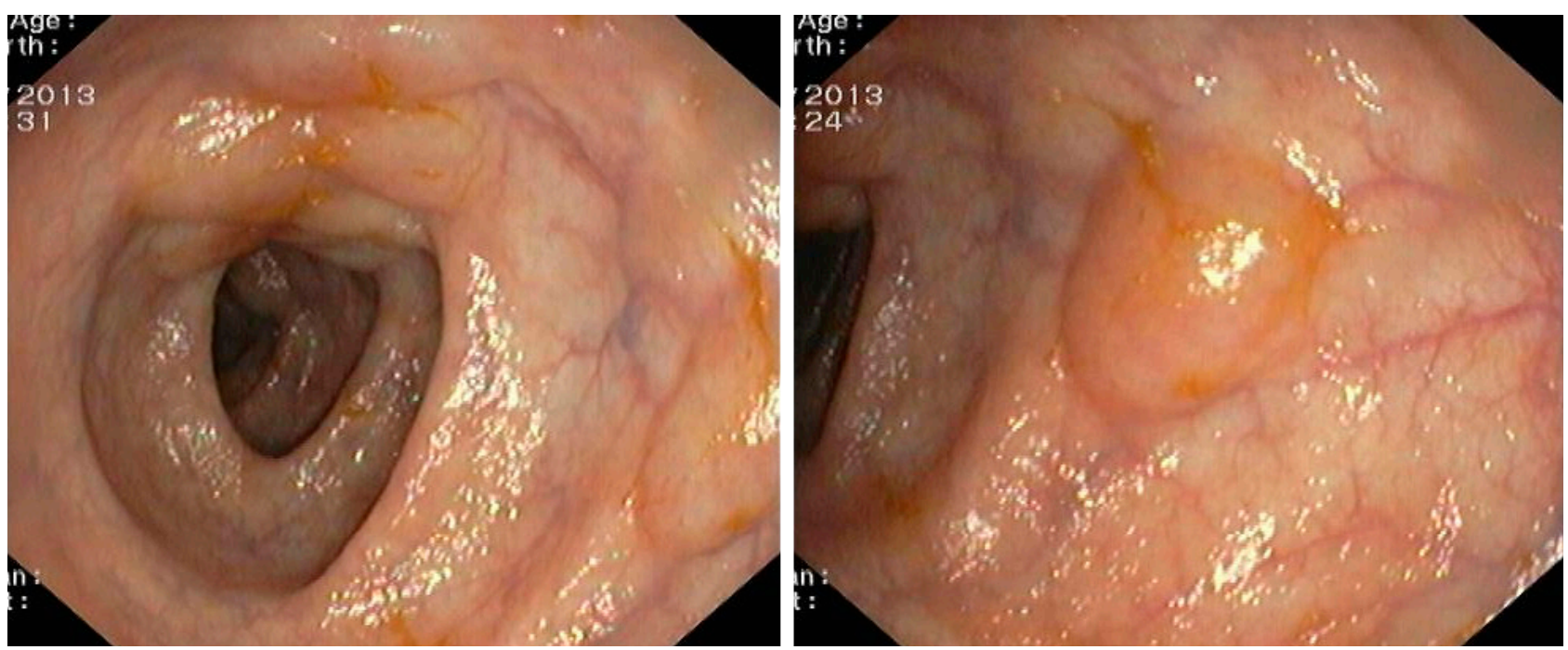

Figura 1 - Aspeto endoscópico da pneumatose cólica da doente descrita em que são evidentes elevações nodulares subepiteliais cobertas por mucosa de aspeto normal

1. Serviço de Gastrenterologia. Centro Hospitalar de Lisboa Central. Lisboa. Portugal.

2. Serviço de Cirurgia Geral. Centro Hospitalar de Lisboa Central. Lisboa. Portugal.

3. Centro de Medicina Subaquática e Hiperbárica. Lisboa. Portugal.

$\triangle$ Autor correspondente: Mariana Costa. mariananunocosta@gmail.com

Recebido: 26 de Dezembro de 2014 - Aceite: 26 de Março de 2015 | Copyright $\odot$ Ordem dos Médicos 2015 


\section{CASO CLÍNICO}

Mulher, 59 anos, com enfartamento, distensão abdominal e hematoquézia ocasional nos últimos seis meses. Antecedentes pessoais de diabetes mellitus, hipertensão arterial e síndrome depressivo, estando medicada com irbesartan + hidroclorotiazida, metformina + sitagliptina, escitalopram e zolpidem. Negava história familiar de cancro colorectal e cirurgias prévias. O abdómen apresentava-se globoso, indolor à palpação, sem tumores nem organomegalias palpáveis. Ao exame proctológico, pele da região perianal sem lesões. Toque rectal indolor, esfíncter normotónico, não se palpando massas ou áreas deprimidas. Sem sangue na luva. Analiticamente apresentava proteína $C$ reativa $23,9 \mathrm{mg} / \mathrm{L}$ com leucócitos e hemoglobina dentro dos valores de referência. Realizou colonoscopia observando-se múltiplas elevações nodulares subepiteliais de superfície lisa, cobertas por mucosa de aspeto normal, desde a sigmoide ao cego. As lesões colapsavam durante as biópsias, surgindo ar (Fig. 1). A histologia confirmou a PI.

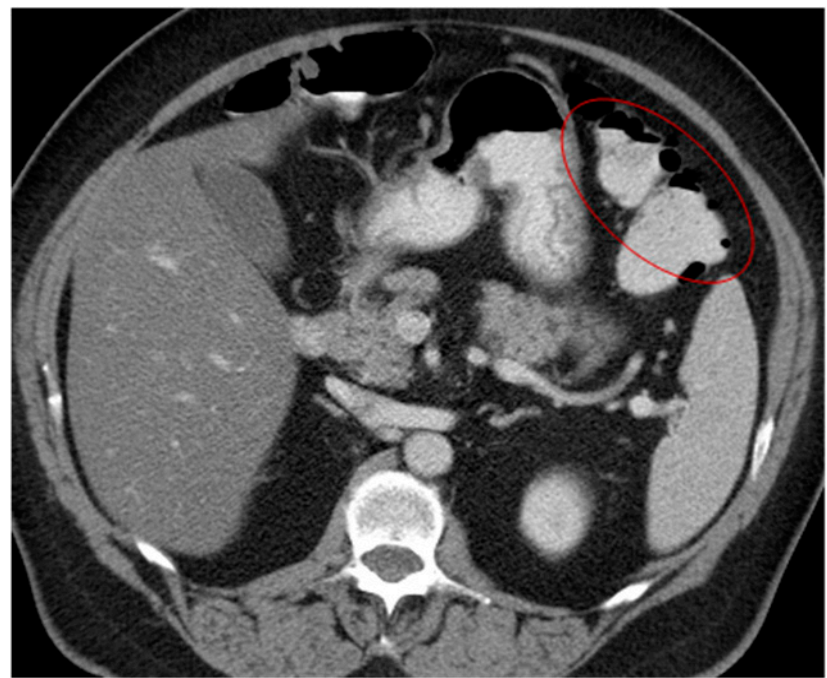

Figura 2 - TC abdominal após cirurgia evidenciando imagens gasosas quísticas no ângulo esplénico do cólon
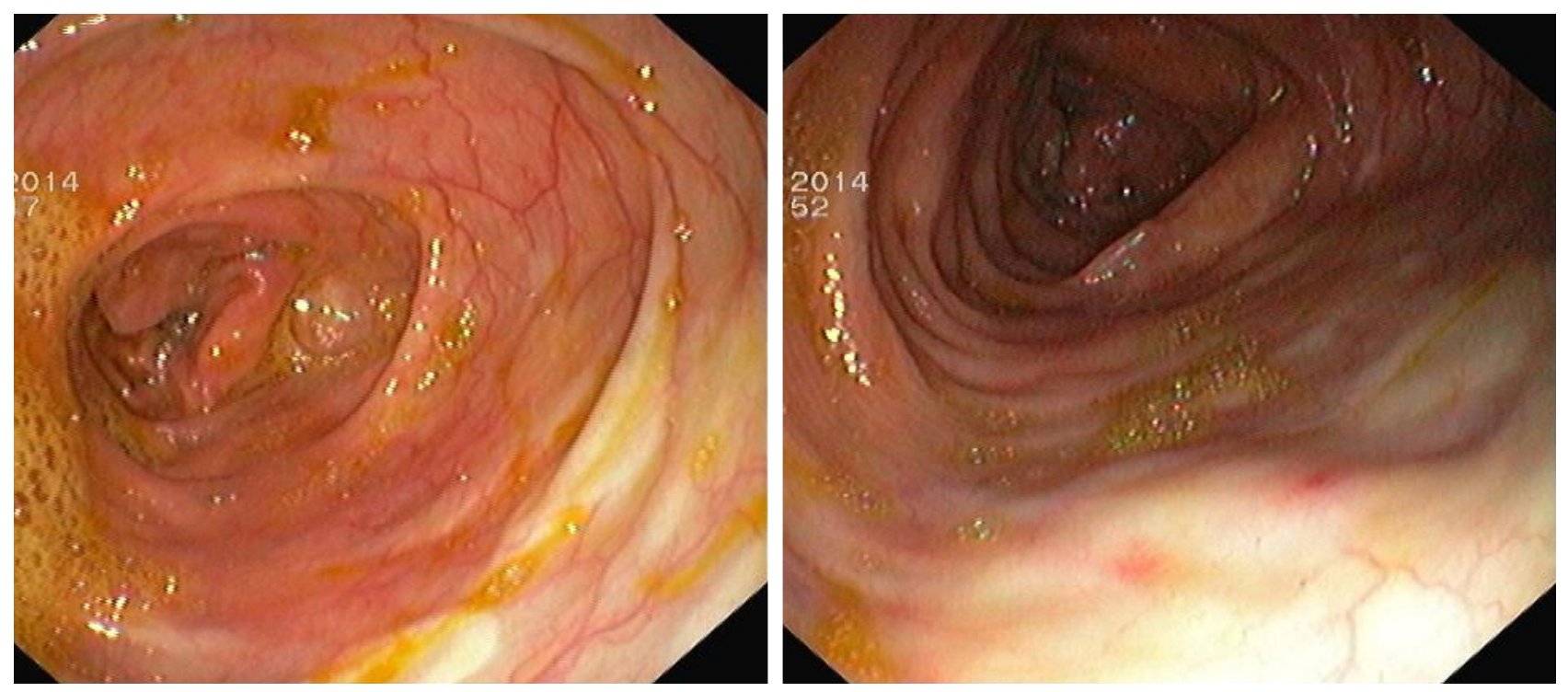

A TC abdomino-pélvica evidenciou lesão ocupando espaço, sólida, na área anexial direita, medindo cerca de $10 \times 7,5$ $\mathrm{cm}$ e, no cólon ascendente e transverso, imagens quísticas intramurais; contudo sem sinais de perfuração intestinal. A doente foi submetida a histerectomia total, anexectomia bilateral e apendicectomia. $\mathrm{O}$ estudo anatomo-patológico identificou teratoma benigno do ovário direito. Após a cirurgia manteve distensão abdominal. Realizou TC abdomino-pélvica que documentou manutenção de pequenas imagens quísticas na parede do ângulo esplénico do cólon (Fig. 2). Iniciou terapêutica com metronidazol (500 mg 3 id per os) e OHB - 2,5 atmosferas absolutas (ATM) durante 90 minutos, 5 dias por semana. Findo cada ciclo de 20 sessões foi reavaliada clinicamente. Cumpriu um total de 80 sessões, após as quais ocorreu melhoria sintomática. No final do tratamento a colonoscopia mostrou, no cólon ascendente e transverso, áreas de mucosa esbranquiçada (Fig. 3). O exame histológico evidenciou fibrose do córion. A TC não identificou sinais de PC.

\section{DISCUSSÃO}

A PI é uma entidade rara e ainda pouco compreendida. Não se trata de uma verdadeira doença mas de um sinal clínico que está associado a uma patologia subjacente em $85 \%$ dos casos (Tabela 1 ). ${ }^{2}$ A sua incidência é difícil de determinar porque muitos doentes são assintomáticos. Pode ocorrer em crianças e adultos, principalmente entre a quinta e oitava décadas de vida, com incidência semelhante em ambos os sexos. ${ }^{3}$ Têm sido propostas várias hipóteses para explicar a sua fisiopatologia: mecânica, bacteriana e bioquímica; que apesar de díspares, não são mutuamente exclusivas. De acordo com a teoria mecânica, o gás disseca a parede intestinal através de disrupções da mucosa ou através da serosa a partir dos vasos mesentéricos. ${ }^{4} \mathrm{~A}$ teoria bacteriana advoga que as bactérias formadoras de gás terão acesso à submucosa através de pontos de rotura da mucosa. ${ }^{5}$ Finalmente, a hipótese bioquímica propõe que as bactérias luminais produzam grandes quantidades de

Figura 3 - Colonoscopia após tratamento: Cicatrizes no cólon ascendente e transverso. 
Tabela 1 - Doenças associadas à pneumatose intestinal ${ }^{14-15}$

Abdómen agudo

Disrupção da mucosa

Infecções

Distúrbios pulmonares

Procedimentos endoscópicos

Doenças que afectam a motilidade GI

Distúrbios imunológicos
Isquemia intestinal, enfarte intestinal, perfuração intestinal, enterocolite necrotizante, tiflite

Doença ulcerosa péptica, doença de Crohn, colite ulcerosa, jejunostomia de alimentação, ingestão de cáusticos, rotura de divertículo

Clostridium difficile, tuberculose, doença de Whipple, enterocolite associada à SIDA (Cryptosporidium, Mycobacterium avium, citomegalovírus)

Doença pulmonar obstrutiva crónica, asma, fibrose quística, ventilação mecânica

Endoscopia digestiva alta, colonoscopia, escleroterapia, colangiopancreatografia retrógrada endoscópica

Diabetes Mellitus, esclerodermia, doença de Hirschprung, pseudo-obstrução intestinal, bypass jejuno-ileal, obstrução/estenose pilórica

Síndrome de imunodeficiência adquirida, corticóides, quimioterapia, doenças linfoproliferativas, transplante de medula óssea, transplante de órgãos sólidos, doença do enxerto versus hospedeiro, amiloidose, doença vascular do colagénio hidrogénio aumentando a sua pressão no interior do lúmen intestinal, forçando-o a atravessar diretamente a mucosa, ficando sequestrado na submucosa. ${ }^{6}$ Nenhuma das diferentes teorias consegue explicar o processo fisiopatológico por completo. No caso apresentado considerou-se que a compressão pelo teratoma ovárico tenha aumentado a pressão gasosa no cólon e induzido a formação quística.

A maioria dos doentes é assintomática. Todavia, diarreia, hematoquézia, desconforto abdominal, distensão abdominal, obstipação, perda ponderal e tenesmo ${ }^{1}$ podem estar presentes. Os sintomas podem ainda estar associados à patologia de base. Complicações, como oclusão intestinal, volvo, invaginação, pneumoperitoneu e hemorragia, ocorrem em aproximadamente $3 \%$ dos doentes. ${ }^{7}$

O diagnóstico baseia-se em técnicas de imagem. A TC é o método mais sensível e pode identificar a patologia subjacente e complicações associadas. Os achados característicos incluem coleções de ar na parede intestinal sem o nível hidroaéreo característico do ar intraluminal. $\mathrm{Na}$ doente apresentada, e dada a clínica, a colonoscopia foi o primeiro exame realizado, indiciando o diagnóstico.

A terapêutica da PI é direcionada para a patologia subjacente. Se o doente mantiver sintomas, como no caso exposto, estão indicadas outras opções terapêuticas como antibióticos, em particular o metronidazol - eficaz contra bactérias anaeróbias, ${ }^{8}$ dieta elementar, cuja eficácia está presumivelmente associada à alteração da flora intestinal ${ }^{9}$; oxigenoterapia normo ou hiperbárica - além de ser tóxica para as bactérias anaeróbias, a elevada concentração de oxigénio no sangue aumenta a sua pressão parcial e diminui a pressão parcial dos restantes gases criando um gradiente de difusão através da parede dos quistos ${ }^{10}$; ressecção cirúrgica da área intestinal afetada (principalmente se houver oclusão intestinal) ${ }^{11}$; ou terapêutica endoscópica através de punção e escleroterapia dos quistos, nos doentes com sintomas obstrutivos e sem condições cirúrgicas. ${ }^{12}$ O tratamento mais eficaz é a aliança terapêutica. No caso descrito optou-se pelo tratamento com metronidazol associado a OHB. O metronidazol é o antibiótico mais amplamente utilizado no tratamento da $\mathrm{PI}$, apesar da pouca evidência científica para a sua utilização. ${ }^{8}$

A OHB tem sido reconhecida como uma terapêutica eficaz na $\mathrm{PI}$, que está incluída na listagem das recomendações do European Committee for Hyperbaric Medicine. ${ }^{13}$ Apesar de não existirem ensaios clínicos randomizados, numa revisão recente de Feuerstein JD et al, ${ }^{7}$ identificaram-se 35 casos cujo tratamento foi a $\mathrm{OHB}$. Obteve-se resolução ou melhoria sintomática em $89 \%$ dos doentes. $O$ protocolo terapêutico utilizado (nível de ATM, tempo de tratamento e duração global do mesmo) foi variável consoante os relatos. Não houve registo de complicações.

Segundo a literatura, a OHB parece ser um método seguro e eficaz no tratamento da PI. No caso apresentado houve resolução completa das lesões e melhoria sintomática com terapêutica combinada com metronidazol e OHB, sem efeitos adversos.

\section{PROTECÇÃO DE PESSOAS E ANIMAIS}

Os autores declaram que os procedimentos seguidos estavam de acordo com os regulamentos estabelecidos pelos responsáveis da Comissão de Investigação Clínica e Ética e de acordo com a Declaração de Helsínquia da Associação Médica Mundial.

\section{CONFIDENCIALIDADE DOS DADOS}

Os autores declaram ter seguido os protocolos do seu centro de trabalho acerca da publicação dos dados de doentes. 


\section{CONFLITOS DE INTERESSE}

Os autores declaram que não houve conflitos de interesse na realização deste trabalho.

\section{REFERÊNCIAS}

1. Jamart J. Pneumatosis cystoides intestinalis. A statistical study of 919 cases. Acta Hepatogastroenterol. 1979;26:419.

2. Koss LG. Abdominal gas cysts (pneumatosis cystoides intestinorum hominis); an analysis with a report of a case and a critical review of the literature. AMA Arch Pathol. 1952;53:523-549.

3. Knechtle SJ, Davidoff AM, Rice RP. Pneumatosis intestinalis. Surgical management and clinical outcome. Ann Surg 1990; 212:160-5.

4. Pieterse AS, Leong AS, Rowland R. The mucosal changes and pathogenesis of pneumatosis cystoides intestinalis. Hum Pathol. 1985;16:683.

5. Yale CE, Balish E, Wu JP. The bacterial etiology of pneumatosis cystoides intestinalis. Arch Surg. 1974;109:89.

6. Sartor RB, Murphy ME, Rydzak E. Miscellaneous inflammatory and structural disorders of the colon. In: Yamada T, Alpers DH, Laine L, Owyang C, Powell DW, editors. Textbook of Gastroenterology. $3^{\text {rd }}$ ed. Philadelphia: Lippincott Williams \& Wilkins; 1999. p. 409-14.

7. Feuerstein JD, White N, Berzin TM. Pneumatosis intestinalis with a focus on hyperbaric oxygen therapy. Mayo Clin Proc. 2014;89:697-703.

8. Ellis BW. Symptomatic treatment of primary pneumatosis coli with metronidazole. Br Med J. 1980;280:763.

\section{FONTES DE FINANCIAMENTO}

Os autores declaram que não houve subsídio(s) ou bolsa(s) que tenham contribuído para a realização do trabalho.

9. Johnston BT, McFarland RJ. Elemental diet in the treatment of pneumatosis coli. Scand J Gastroenterol. 1995;30:1224.

10. Levitt MD, Olsson S. Pneumatosis cystoides intestinalis and high breath $\mathrm{H} 2$ excretion: insights into the role of $\mathrm{H} 2$ in this condition. Gastroenterology. 1995;108:1560.

11. Greenstein AJ, Nguyen SQ, Berlin A, Corona J, Lee J, Wong E, et al. Pneumatosis intestinalis in adults: management, surgical indications, and risk factors for mortality. J Gastrointest Surg. 2007;11:1268.

12. Johansson $\mathrm{K}$, Lindström $\mathrm{E}$. Treatment of obstructive pneumatosis coli with endoscopic sclerotherapy: report of a case. Dis Colon Rectum. 1991;34:94.

13. European Committee for Hyperbaric Medicine. Recommendations of the jury of the 7th European Consensus Conference on Hyperbaric Medicine, Lille, 2004; [consultado 2014 Dez 21]. Disponível em: http://www.echm. org/ECHM-Conferences.htm.

14. Koss LG. Abdominal gas cysts (pneumatosis cystoides intestinorum hominis); an analysis with a report of a case and a critical review of the literature. AMA Arch Pathol. 1952;53:523

15. Knechtle SJ. Pneumatosis intestinalis. Surgical management and clinical outcome. Ann Surg. 1990;212:160. 
Mariana COSTA, Carolina MORGADO, David ANDRADE, Francisco GUERREIRO, João COIMBRA

\section{Pneumatose Cólica Tratada com Metronidazol e Oxigenoterapia Hiperbárica: Um Caso de Sucesso}

Acta Med Port 2015:28:534-537

Publicado pela Acta Médica Portuguesa, a Revista Científica da Ordem dos Médicos

Av. Almirante Gago Coutinho, 151

1749-084 Lisboa, Portugal.

Tel: +351218428 215

E-mail: submissao@actamedicaportuguesa.com

www.actamedicaportuguesa.com

ISSN:0870-399X | e-ISSN: 1646-0758

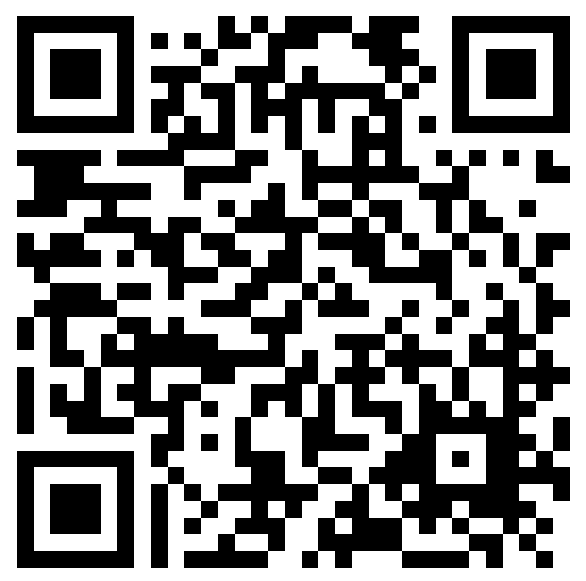

